\title{
Staphylococcal Pyomyositis Within Initial Course of Juvenile Dermatomyositis Patient
}

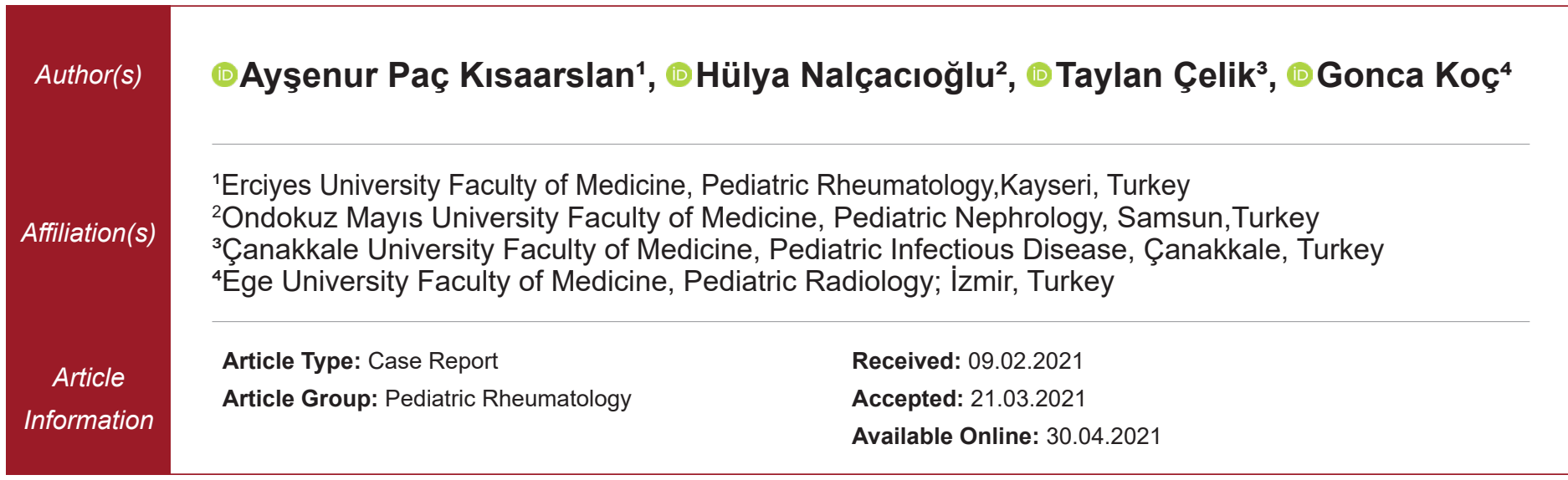

Cite this article as: Paç Kısaarslan A, Nalçacıoğlu H, Çelik T, Koç G. Staphylococcal Pyomyositis Within Initial Course of Juvenile Dermatomyositis Patient. J Pediatr Acad 2021; 2: 36-38.

\section{Abstract}

Infectious complications are increasingly reported in patients with connective tissue diseases. These complications are both related to using immunosuppressive drugs and organ dysfunctions caused by diseases. Pyomyositis is a pyogenic infection of skeletal muscle associated with immunocompromised conditions, especially human immunodeficiency virus (HIV) infection, diabetes mellitus, malignancy, immunosuppressive drugs, and rheumatic diseases. We presented a patient with juvenile dermatomyositis complicated by staphylococcal pyomyositis initial disease course. The patient did not respond to drainage and appropriate antibiotic therapy. Abscess formation successfully regressed with IVIG. Muscle inflammation and corticosteroids are possible predisposing factors for pyomyositis.

Keywords: Dermatomyositis, pyomyositis, treatment

\section{Introduction}

Pyomyositis is a pyogenic infection of skeletal muscle that rises from the hematogenous spread and usually presents with localized abscess. ${ }^{1}$ It is associated with immunocompromised conditions, especially human immunodeficiency virus (HIV) infection, diabetes mellitus, malignancy, immunosuppressive drugs, and rheumatic diseases. ${ }^{2,3}$
Dermatomyositis (DM)/Polymyositis (PM) are systemic inflammatory disorders affecting skeletal muscles and other organs. ${ }^{4}$ Juvenile DM diagnosis is based on the 2017 European League Against Rheumatism/American College of Rheumatology (EULAR/ACR) classification criteria. ${ }^{5}$ This classification system involves age, symmetric proximal muscle weakness, characteristic skin changes:

Correspondence: Ayşenur Paç Kısaarslan, Erciyes University Faculty of Medicine, Pediatric 
heliotrope rash, Gottron sign and papules, elevation muscle enzymes, electromyographic changes, magnetic resonance, and muscle biopsy findings. ${ }^{5,6}$ Polymyositis is uncommon in children and is defined by the absence of characteristic skin changes. ${ }^{5,6} \mathrm{DM} / \mathrm{PM}$ is considered to be associated with high morbidity and mortality rates, in some cases as high as $50 \%$, primarily related to life-threatening muscle weakness, cardiac and lung impairment, as well as infectious manifestations. Infectious complications that involved pyogenic and opportunistic infections have been described in $26-37.3 \%$ of DM/PM patients. ${ }^{4}$ Several factors may be implicated in this apparent such as increased frequency of infections in DM/PM patients, particularly immunosuppressive medications. Due to DM/PM itself, immune system dysfunction may lead to elevated susceptibility to opportunistic infections. Three cases of pyomyositis with DM were reported in the literature since 1990's. ${ }^{7-9}$

Here we presented a patient with juvenile dermatomyositis complicated of staphylococcal pyomyositis after the initial disease course.

\section{Case}

A four-year-old girl was admitted to the hospital because of severe pain on extremities after an upper airway infection. She was diagnosed with post-infectious myositis and prescribed ibuprofen therapy. After ten days, her pain persisted, and she was referred to our rheumatology clinic with the chief complaint of severe pain, edema on extremities, and disabling walking. Upon presentation, she was afebrile with no other constitutional symptoms. On the physical examination, malar and heliotropic rash on her face, vasculitic rash on plantar faces of hands and feet, severe nonpitting edema on extremities were detected. Muscle strength was not determined because of the severe pain. Haemogram and blood smear were normal, CRP: $12.5 \mathrm{~g} / \mathrm{L}$, Erythrocyte Sedimentation Rate: 19 mm/h, AST: 42 IU/L, ALT: 80 IU/L; LDH:1007 IU/L, CK: $500 \mathrm{IU} / \mathrm{L}$, immunoglobulins, C3, and C4 were normal, ANA:-, ANA subgroups:-, vWFAg: 183.7 (high level) were detected. EBV, CMV, Parvovirus, HIV, and hepatitis B, C serology were negative. A severe hyperintense signal at all of the girdle muscle on T2 weighted images was detected (Figure 1). Our patient was diagnosed with JDM based on the heliotrope rash, symmetric proximal muscle weakness, elevated muscle enzymes, and MRI findings. Her total diagnostic score was 9.4 according to the 2017 EULAR/ACR classification critera. ${ }^{5}$ She was started prednisolone $2 \mathrm{mg} /$ $\mathrm{kg} / \mathrm{d}$ and methotrexate $15 \mathrm{mg} / \mathrm{m}^{2} / \mathrm{w}$. The CMAS (Childhood Myositis Assessment Scale, an assessment of muscle strength and the total score is 52), was detected 28 on the therapy's $5^{\text {th }}$ day. After ten days of immunosuppressive therapy, we noticed localized tenderness, erythema, and swelling on the left gluteal side. An abscess formation was determined as $24^{\star} 37^{\star} 45 \mathrm{~mm}$, and it was lied down posterior to the inferior of gluteus maximus muscle by repeated MRI (Figure 2). The pus was drained through guided needle aspiration on the first day of hospitalisation. A gram-stained preparation showed gram-positive cocci in clusters. The culture of the pus yielded methicillin-sensitive $S$. aureus. After 2 days later, clindamycin and gentamycin were added to ceftriaxone. The abscess formation was not regressed through a drainage tube and appropriate antibiotic therapy on the $14^{\text {th }}$ day. IVIG, which was recommended for dermatomyositis therapy also, was infused as $1 \mathrm{gr} / \mathrm{kg}$. The patient improved two days later, steroid and methotrexate therapies were continued. The abscess formation did not repeat.

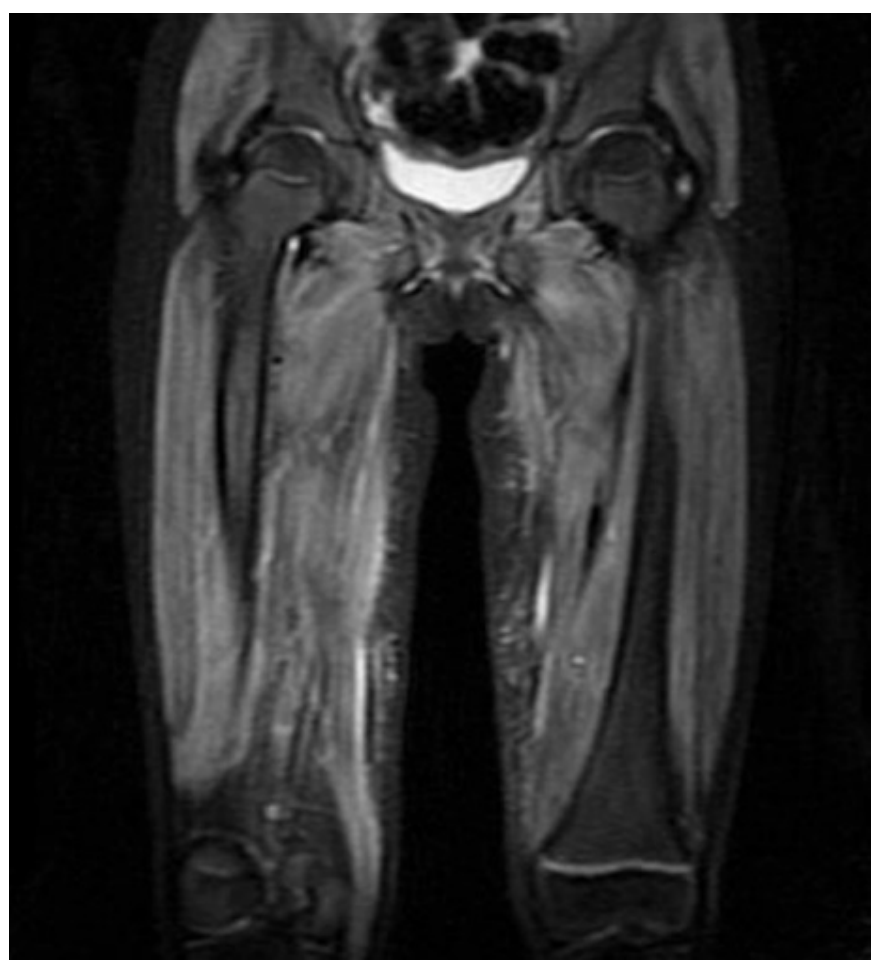

Figure 1. Hyperintense signal at all of the girdle muscle on T2 weighted images on MRI.

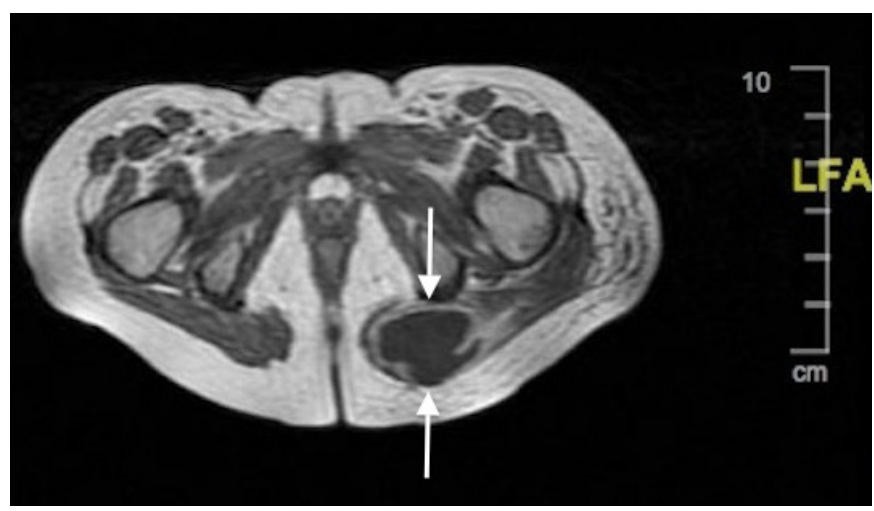

Figure 2. An abscess formation were determined as $24^{*} 37^{*} 45 \mathrm{~mm}$, and it was lied down posterior to inferior of gluteus maximus muscle by MRI.

\section{Discussion}

We presented a patient with dermatomyositis complicated of staphylococcal pyomyositis initial course of the disease.

Infectious complications are increasingly reported in connective tissue diseases. These are related to using immunosuppressive drugs and is related to organ dysfunctions caused by diseases. Marie et al. ${ }^{4}$ reported that infectious complications had been described $37.3 \%$ in $279 \mathrm{DM} / \mathrm{PM}$ patients. The most common pyogenic infections are aspiration pneumonia and calcinosis cutis infections. ${ }^{4}$ 
Most of the reports on pyomyositis were in the 1990s and mostly reported from tropical areas, named "Tropical myositis". In recent years, PM has been more frequently diagnosed in immunocompetent children presenting with limping, even in temperate countries..$^{10}$ Infectious myopathies are mostly associated with immunocompromised conditions, especially HIV infection, diabetes mellitus, malignancy, immunosuppressive drugs, and rheumatic diseases. ${ }^{11}$

The clinical presentation of pyomyositis can be divided into three stages. The first stage is typically subacute, occurring over 1 to 3 weeks, with local swelling and "woody" texture, mild pain, and variable fevers. The diagnosis is often confused with thrombosis, hematoma, contusion, muscle strain, or osteomyelitis because the purulent collection has not yet developed. During the second or suppurative stage, which occurs at 10 to 21 days, tenderness and fevers become more pronounced. The diagnosis of pyomyositis is usually established in this stage. If the infection remains undiagnosed and untreated, intense local pain and fluctuance, systemic findings, sepsis will develop as part of the third stage of pyomyositis. ${ }^{12}$

In our patient, abscess formation was detected approximately 25 days after the onset of generalize muscle pain and ten days after immunosuppressive treatment. In the first MRI findings did not show abscess (Figure 1). Attempts to create pyomyositis in animal muscles challenged with intravenous injection of $S$. aureus failed to initiate the infection unless the muscles were traumatized by electric shock, pinching, or ischemia. These experiments confirm the skeletal muscle's high intrinsic resistance to bacterial infection and suggest that underlying muscle damage facilitates its onset..$^{13}$ Although there was no injection or trauma history, muscle inflammation and immunosuppressive therapy may have facilitated abscess formation in our patient.

The majority of cultures with infectious myositis were yielded $S$. aureus. Increased staphylococcal infections in childhood dermatomyositis were previously reported associated with calcinosis. We founded staphylococcal pyomyositis in one patient, streptococcal pyomyositis in two patients with $\mathrm{DM}$ in the literature ${ }^{7-9}$ At last, Tuberculous pyomyositis was reported in SLE and DM patients in 2014. ${ }^{14}$

We present a patient with pyomyositis involved in the differential diagnosis of JDM. We think that pyomyositis developed as a complication in this case. JDM presents with clinically symmetrical proximal muscle weakness and muscle pain. However, pyomyositis should be kept in mind in the presence of local pain and other signs of inflammation.
Author Contributions: APK: Concept and designed the study, Wrote the manuscript. HN: Collected the data and helped the writing; TÇ: Collected the data; GK: Collected the data (All of the authors declare that they have all participated in the design, execution, and analysis of the paper, and that they have approved the final version)

Conflict of Interest: There are no conflicts of interest in connection with this paper, and the material described is not under publication or consideration for publication elsewhere.

Financial Disclosure: The authors declared that this study has received no financial support

Informed Consent: Written informed consent was obtained from patients who participated in this study.

Peer-review: Externally peer-reviewed.

\section{References}

1. Lopez FA, Lartchenko S. Skin and soft tissue infections. Infect Dis Clin North Am. 2006;20:759-vi. [CrossRef]

2. Mitsionis GI, Manoudis GN, Lykissas MG, et al. Pyomyositis in children: early diagnosis and treatment. $J$ Pediatr Surg. 2009;44:2173-2178. [CrossRef]

3. Blay G, Ferriani MP, Buscatti IM, França CM, Campos LM, Silva CA. Pyomyositis in childhood-systemic lupus erythematosus. Rev Bras Reumatol Engl Ed. 2016;56:79-81. [CrossRef]

4. Marie I, Ménard JF, Hachulla E, et al. Infectious complications in polymyositis and dermatomyositis: a series of 279 patients. Semin Arthritis Rheum. 2011;41:48-60. [CrossRef]

5. Bottai M, Tjärnlund A, Santoni G, et al. EULAR/ACR classification criteria for adult and juvenile idiopathic inflammatory myopathies and their major subgroups: a methodology report. RMD Open. 2017;3:e000507. [CrossRef]

6. Petty RE. Textbook of Pediatric Rheumatology. 8th ed. Philadelphia: Elsevier 2021:360-377.

7. Gelfand MS, Holladay R, Bertorini T, Adams RF. Staphylococcal pyomyositis with idiopathic dermatomyositis. J Tenn Med Assoc. 1989;82:243-244. [CrossRef]

8. Casademont J, Roger N, Pedrol E, García F, Herrero C, Grau JM. Streptococcal myositis as a complication of juvenile dermatomyositis. Neuromuscul Disord. 1991;1:375-377. [CrossRef]

9. Soriano ER, Barcan L, Clara L, Imamura P, Catoggio LJ. Streptococcus pyomyositis occurring in a patient with dermatomyositis in a country with temperate climate. J Rheumatol. 1992;19:1305-1307. [CrossRef]

10. Amoozgar B, Kaushal V, Garsondiya B. Primary Pyomyositis: Contact Sports as the Rare Risk Factors. Case Rep Infect Dis. 2019;2019:5739714. [CrossRef]

11. Fox LP, Geyer AS, Grossman ME. Pyomyositis. J Am Acad Dermatol. 2004;51:308-314. [CrossRef]

12. Crum-Cianflone NF. Bacterial, fungal, parasitic, and viral myositis. Clin Microbiol Rev. 2008;21:473-494. [CrossRef]

13. Christin L, Sarosi GA. Pyomyositis in North America: case reports and review. Clin Infect Dis. 1992;15:668-677. [CrossRef]

14. Simopoulou T, Varna A, Dailiana $Z$, et al. Tuberculous pyomyositis: a re-emerging entity of many faces. Clin Rheumatol. 2016;35:11051110. [CrossRef] 